\title{
TEST OF A BOUNCER MODULATOR WITH PULSE CABLE AT FLASH
}

\author{
H.-J. Eckoldt, N. Ngada, J. Havlicek, A. Hauberg, S. Choroba, \\ T. Grevsmuehl, I. Sokolov, T. Froelich \\ DESY, Germany
}

Keywords: Pulse cable, modulator, high power pulses, XFEL, free electron laser.

\begin{abstract}
The superconducting XFEL machine will be built in a single tunnel. The RF stations provide RF power of up to $10 \mathrm{MW}$ at $1.3 \mathrm{GHz}$. The modulators will produce high-voltage pulses of up to $120 \mathrm{kV}, 140 \mathrm{~A}$ with a pulse length of $1.54 \mathrm{~ms}$. These will be installed in a service hall at ground level. The klystrons will be positioned near the cavities inside of the tunnel. The energy will be transported via pulse cables of up to $1.5 \mathrm{~km}$ length between the pulse generating units of the modulators located in the halls and the pulse transformers in the tunnel. Since the transformers have a transformer ratio of $1: 12$, pulses of $10 \mathrm{kV}$ and $1.6 \mathrm{kA}$ must be transmitted. Tests with pulse cables have been made with an existing modulator and klystron of the FLASH-Linac. The results showed that the internal construction of the modulator had to be changed to improve EMI behaviour. In order to prove that the beam in the accelerator is not disturbed by the operation of the cables a set of pulse cables was installed inside the tunnel of the FLASH-Linac in parallel to the beam line. An existing pulse transformer/ klystron unit was supplied via these $1.5 \mathrm{~km}$ long cables by a new prototype of a modulator taking into account EMI considerations. The FLASH-Linac has been operated for a longer period of time with this set up proving reliable and stable operation.
\end{abstract}

\section{Introduction}

In the superconducting XFEL (X-Ray Free Electron Laser) the $\mathrm{RF}$ is generated in klystrons that are installed inside the accelerator tunnel. The modulators that are supplying the klystrons will be positioned in a central modulator building. This solution was chosen in order to have the possibility of repairing and maintaining modulators during the operation of the XFEL. Via pulse cables the high power pulses are transported into the tunnel. The R\&D for this cable was done and a prototype of the cable was designed, manufactured and commissioned in the FLASH accelerator.

With the results of the first test series improvements in the modulators were defined and a new prototype of modulator was built. In a second test series the combination of new modulator, pulse cable and klystron with transformer was investigated.

\section{Pulse cable}

The pulse cables are of tri-axial design. This construction was chosen since the magnetic fields due to the currents are compensated. The inner and the middle conductors building a coaxial arrangement are used to carry the high power pulses. The shield consists of a solid copper foil. It has several functions. These are the safety shield for high voltage, shield against EMI and it is part of the fire resistance capability of the cable.

Simulations done with Simplorer and pSpice [1] as well as analytic calculations [3] showed that by paralleling cables the increase in rise time due to the cable inductance can be minimized. The overall cross section of the system-cable is defined to be $300 \mathrm{~mm}^{2}$ for the use of the $10 \mathrm{MW}$-klystron In order to match the klystron impedance either three cables will be used for the $5 \mathrm{MW}$-klystron or four cables will be used for the $10 \mathrm{MW}$-klystron of the XFEL. For the tests at FLASH a 5 MW-klystron was used.

The cross section for one cable was chosen to be $75 \mathrm{~mm}^{2}$ for the inner and middle lead. The cross section of the shield was defined to be $16 \mathrm{~mm}^{2}$. With this design it is possible to produce the cable on a standard production line of a cable manufacturer. A sample of the pulse cable can be seen in Fig.1.

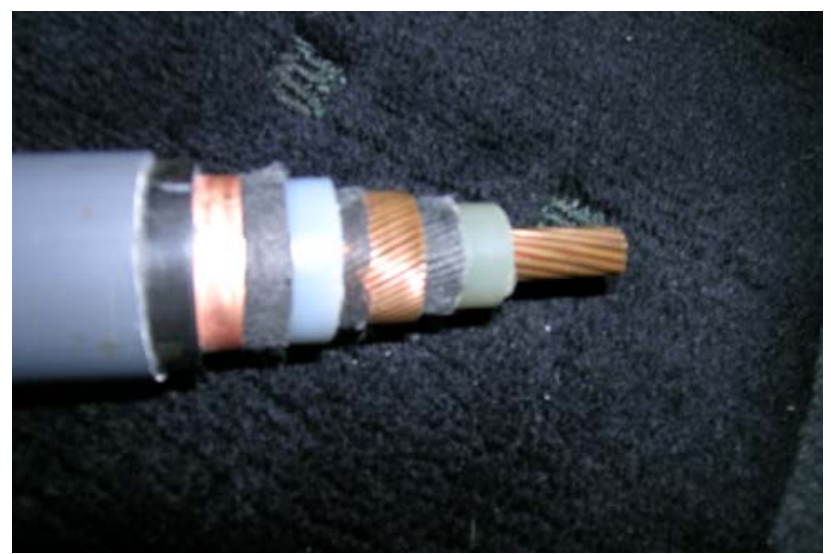

Fig.1 Pulse cable

The cable data is shown in Tab. 1. These are in good match with the theoretical values that can be derived from the mechanical dimensions. It has to be taken into account that the inductance has to be calculated with Eq.1. 


$$
L=\frac{\mu_{0}}{2 \pi} \ln (D / d+0.25)
$$

with

$\mathrm{D}=$ inner diameter of the outer copper lead

$\mathrm{d}=$ diameter of the inner copper lead

This formula is valid for low frequencies and is a suitable approximation for this pulsed application [9]. A detailed calculation was done in [5].

\begin{tabular}{|l|l|l|l|}
\hline & One cable & three cables & Four cables \\
\hline Inductance & $194 \mathrm{nH} / \mathrm{m}$ & $65 \mathrm{nH} / \mathrm{m}$ & $48.5 \mathrm{nH} / \mathrm{m}$ \\
\hline Capacitance & $248 \mathrm{pF} / \mathrm{m}$ & $744 \mathrm{pF} / \mathrm{m}$ & $992 \mathrm{pF} / \mathrm{m}$ \\
\hline Resistance & $472 \mu \Omega / \mathrm{m}$ & $157.3 \mu \Omega / \mathrm{m}$ & $118 \mu \Omega / \mathrm{m}$ \\
\hline $\mathrm{Z}$ & $28 \Omega$ & $9.3 \Omega$ & $6.5 \Omega$ \\
\hline $\begin{array}{l}\text { Prop. Speed } \\
\text { theory }\end{array}$ & $144 \mathrm{~m} / \mu \mathrm{s}$ & $144 \mathrm{~m} / \mu \mathrm{s}$ & $144 \mathrm{~m} / \mu \mathrm{s}$ \\
\hline $\begin{array}{l}\text { Prop. Speed } \\
\text { measured }\end{array}$ & $139 \mathrm{~m} / \mu \mathrm{s}$ & $139 \mathrm{~m} / \mu \mathrm{s}$ & $139 \mathrm{~m} / \mu \mathrm{s}$ \\
\hline
\end{tabular}

Tab. 1: Data of the pulse cable

\section{Simulations}

\subsection{Simulation of the power part}

The behaviour of the entire system was simulated with Simplorer. The simulation model is presented in Fig. 2. construction. The enhanced model with three nested transmission lines delivered a very good approximation of the electrical behaviour. The different models represent:

- $\quad$ LTRA1 transmission line formed by the inner to middle lead

- $\quad$ LTRA2 transmission line formed by the middle lead and the shield

- $\quad$ LTRA3 transmission line formed by the shield and the cable tray

\subsection{Investigation of the internal behaviour of the cable}

Additional simulations to investigate the current distribution inside the cable during the pulse were done. The finite element program MAXWELL2D was used and described in [5].

During the pulse skin effects can be seen whereby proximity effects can be neglected. It is important to have a conductor e.g. a copper foil near the cable to carry parasitic currents.

These investigations gave answer to the question whether production tolerances can influence the overall behaviour of the cables. It was shown that the standard production process for the cable is sufficient for the later operation.

\subsection{Adaptation network}

In pulsed applications cables can cause problems due to reflections deriving from the mismatch of impedances at the beginning and the end of the cables. The klystron with the nonlinear impedance as function of the voltage is an

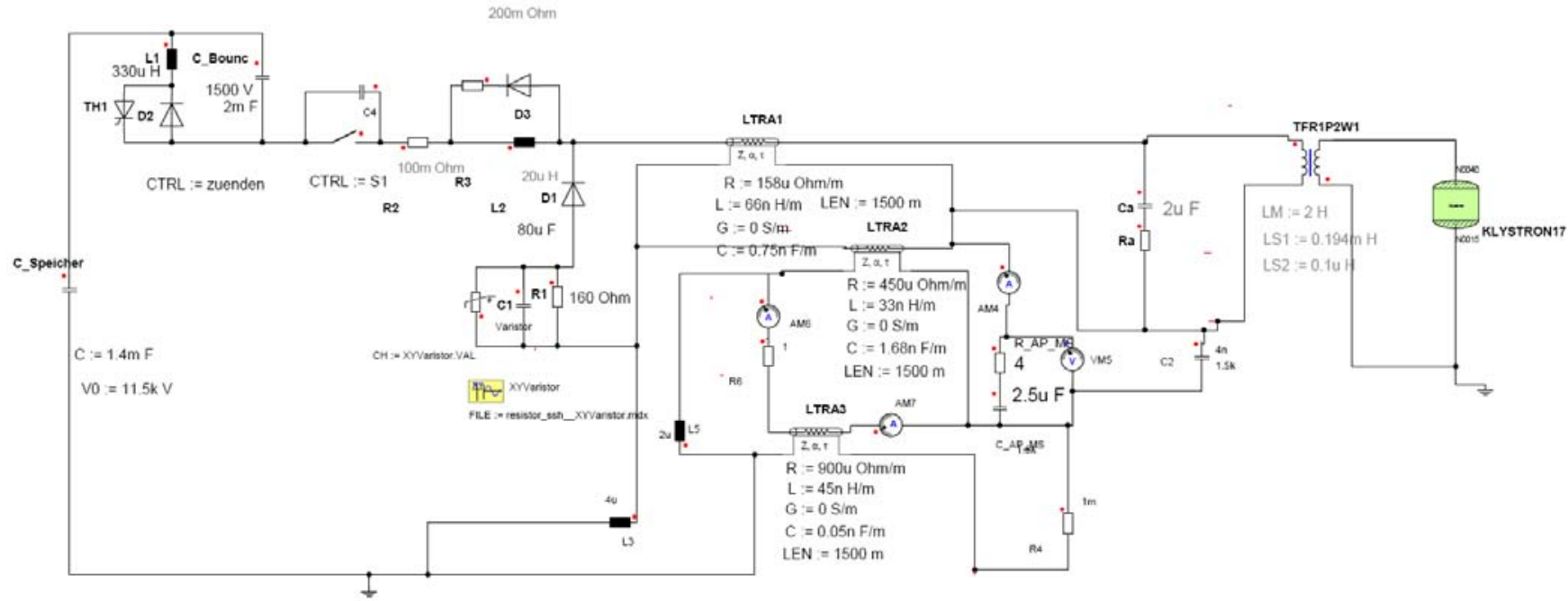

Fig.2: Simulation model

For the klystron a model was developed that represents the nonlinear voltage/current waveform including a short circuit model to investigate klystron arcing. For the simulation of the pulse cable the model for a transmission line with losses was used. The difficulty here was the modelling of the tri-axial additional difficulty. The solution with the pulse cable in combination with fast pulses of the modulator is only possible due to the pulse transformer. With the transformer stray inductance the cable acts electrically like an open ended cable. This can be adapted by a resistance of the same value as the cable impedance $Z_{0}$. Since this resistor shall not build a second load in parallel to the klystron impedance a DC block 
in form of a capacitor has to be introduced. The variation of the capacitance changes the rise time, the voltage overshoot and the losses in the RC network.

When looking at the simulation model it is obvious that all three transmission lines require an adaptation network that is optimized for its $Z_{0}$. The losses in the network are assumed to be less than $5 \mathrm{~kW}$ at the $10 \mathrm{~Hz}$ operation of the linac.

\section{Modulators}

For the first tests the used modulator was of the bouncer type as described in detail in [4,7]. The pulse energy is stored in a capacitor bank at the $10 \mathrm{kV}$ level in order to decouple the pulsed energy from the mains. Via a semiconductor switch this capacitor is connected to the pulse cable and transformer. The transformer has a 1:12 ratio to increase the voltage to the $120 \mathrm{kV}$ level. The current is up to $1680 \mathrm{~A}$ with pulse power up to $16.8 \mathrm{MW}$. The pulse duration for the XFEL will be 1.54 ms. During the pulse the voltage droops for about $19 \%$. To compensate this, a so called bouncer circuit being a LC resonant circuit, is installed. The bouncer circuit is ringing at a frequency of $200 \mathrm{~Hz}$. The output voltage is a superposition of drooping main capacitor voltage and decreasing bouncer sine wave at its most linear section, resulting in a rectangular pulse with a flatness of less than $1 \%$ pk-pk of the pulse amplitude.

\section{First tests till 2004}

As described in [2] the first test have been made in FLASH. A set of four pulse cables in parallel was introduced into an existing set of a modulator, transformer and $5 \mathrm{MW}-\mathrm{klystron.}$ The length of the cable was $1500 \mathrm{~m}$ which corresponds nearly to the longest length that will be used in XFEL. This test environment was sufficient for a proof of principle. It could be verified that pulses of about $10 \mathrm{MW}$ at the $10 \mathrm{kV}$ level can be transported via cables without reflections and significant increase in rise time. The propagation time of the pulse on the cable is $10.8 \mu \mathrm{s}$ which is close to the theoretical value of 10.5 $\mu \mathrm{s}$.

Short circuit tests were performed on a dummy load to prove that the additional energy stored in the cable does not endanger the klystron.

It was proven that the energy transport is feasible but the EMI behaviour of this test set-up was very poor. A lot of ground bouncing occurred due to the fact that the modulator was not designed for the use of the pulse cables. Nearby measurement electronics got disturbed.

Due to the EMI frequency of about $1 \mathrm{MHz}$ measured at the cable, this could be defined to be pure common mode ringing. Disturbances of that frequency in the main path would not be able to leave the cable as explained in $[6,8]$.

The cable continued to be in operation for about $10000 \mathrm{hrs}$. The test modulator and transformer were located next to each other connected to one common ground system. Ground currents easily bypassed the cable. It was not possible to predict what happens when modulator and transformer are separated by a long distance. Therefore it was decided to launch a second test to investigate this in detail.

\section{Test set-up for the second test series}

The second test series had several objectives:

- The new modulator design should suppress the ground bouncing and common mode EMI of the cable.

- The cable was installed along the FLASH accelerator near to the beam to detect whether interferences occur.

- The ground systems of the modulator and the transformer should be separated simulating the situation later in XFEL.

- The pulse cables should be in operation with the FLASH accelerator for a longer period of time to check interferences during the operation.

\subsection{Improvements of the modulators}

A new modulator was built for this test. The bouncer principle with superposing two voltage waveforms was kept. The bouncer LC-circuit however moved to the high potential side. This allows the hard grounding of the return lead and decreases the mechanical dimensions of the return path. The series choke was put at the output. This acts as a dv/dt limiter and blocks high frequency at the output of the power supply. The cabling of the power part was substituted by copper bus bars that were built in a sandwich assembly to decrease parasitic elements formed by the mechanical dimensions. The terminals for connecting the pulse cables were optimized and placed inside of the modulator. The general rise time of the pulse was not influenced by these changes.

\subsection{Installation of the pulse cable}

Again a length of $1500 \mathrm{~m}$ was chosen. Four cables were installed as system-cable for testing both klystron types. The cable was installed on available cable trays at the DESY site, sometimes it had to be installed into earth. Special emphasis was put to the fact that the entire cable tray was connected to give a closed return path. In the places of in-earth installation the cable was wrapped into copper foil to allow parasitic currents to flow. The cable was installed in short distance without additional shielding in parallel to the FLASH accelerator beam line. This was for the entire length of the accelerator of about $200 \mathrm{~m}$. If ever EMI occurred the impact to the beam could have been investigated. The distance between cable and beam was about 80 to $100 \mathrm{~cm}$ as shown in Fig.3.

For this test intersection for the tri-axial cable had been developed, installed and tested successfully.

\subsection{Different grounding systems}

Modulator and transformer were installed in different halls at a distance of about $300 \mathrm{~m}$. Theses halls had mains input from different $10 \mathrm{kV}$ main stations at DESY giving a worst case scenario for the grounding system. 


\section{Test results second test series till 2008}

\subsection{Low frequency ground currents}

Even before starting the operation of the modulator, currents of several amperes with frequencies of $12.5 \mathrm{~Hz}$ and $50 \mathrm{~Hz}$

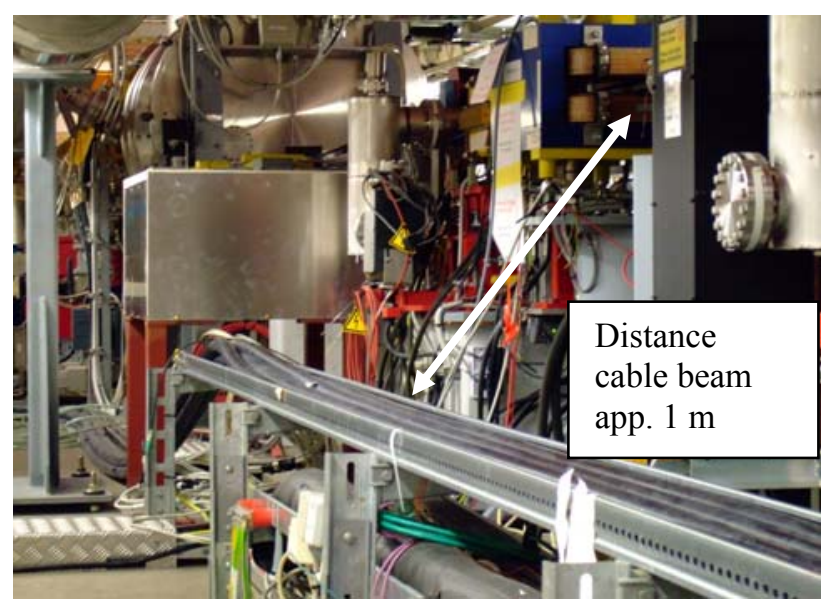

Fig.3 Installation of the cable at a distance of $0.8-1 \mathrm{~m}$

were measured on the shield of the cable. The reason for the $50 \mathrm{~Hz}$ was the potential difference of the two mains systems. The $12.5 \mathrm{~Hz}$ component derived from the operation of the DESYII synchrotron that is operating at this frequency. With the connection of the shield of the cable the two grounding systems where short circuited with a low impedance. The introduction of a resistor of at least $5 \Omega$ in series to the shield at the transformer side solves this problem.

\subsection{EMI behaviour of the new modulator}

The redesign of the modulator was proven to be very effective. The $1 \mathrm{MHz}$ common mode currents at the beginning and the end of the pulse were reduced to less than 2 $\mathrm{A}_{\text {peak }}$. The time duration was less than $20 \mu \mathrm{s}$.

The former high frequency ringing of about $1 \mathrm{MHz}$ had amplitudes of 12 to $15 \mathrm{~A}$.

\subsection{Grounding of the cable}

A standard question on cables is about the grounding of the shield. The cable has to be grounded at the modulator in order to control the currents due to parasitic elements. This is valid for the middle conductor and the shield. At the transformer side different groundings of the shield were tested:

- Transformer side open. This leads to a ringing of the cable with $25 \mathrm{kHz}$ with significant amplitude.

- Grounded on both side. When grounding on both sides common mode current during the pulse did not decrease to zero. Since this current was flowing back outside of the cable this alternative was abandoned in order not to introduce further magnetic fields. As mentioned in 7.1 this is not advisable anyway.

- Transformer side grounded via RC. During the rise time of the pulse it was not possible to get the common mode current down to zero. However it could be decreased to a current less than 2 Amps. After $150 \mu$ s. this had decreased to zero. According to 7.1 at least $5 \Omega$ shall be inserted, $12 \Omega$ seem to be an optimum.

\subsection{Pulse current on the beam line}

Near the gun of FLASH pulse currents were measured in the vacuum chamber over a length of app. $10 \mathrm{~m}$. Tracing down the current path showed that the cables trays where not connected properly in that area. After improving this, the currents in the vacuum chamber disappeared.

\section{Summary}

The modulators of the XFEL shall be installed in a central building for maintenance reasons and radiation protection. Therefore pulse cables have to be introduced. Intense R\&D on cables and modulators was done. It was demonstrated that the pulsed energy can be transported via cables over a distance of $1.5 \mathrm{~km}$. An EMI-optimized modulators was built. FLASH operated for about one year with a set of pulse cables that was introduced into an existing RF-Station proving long term reliability. During another six months operation with the new optimized modulator and a set of pulse cables in parallel and close to the beam line of FLASH no EMI problems occurred.

A future operation of the XFEL with modulators outside of the tunnel and transporting the energy via cables to the transformer-klystron station is feasible.

\section{References}

[1] H-J. Eckoldt, "Pulse Cable for Modulators", TESLAReport 35-2000, DESY

[2] H-J. Eckoldt, "Pulse Cables for XFEL Modulators", LINAC 2006, Knoxville, Tennessee

[3] M. Filtz, "Analytische Berechnungen zu einem Hochspannungskabel", TU Berlin, 2000

[4] W. Kaesler "A long Pulse Modulator for TESLA TEST Facility", $2^{\text {nd }}$ European Pulsed Power Symposium 2004, Hamburg

[5] N. Ngada, "Simulation von Modulatoren mit Pulskabeln unter besonderer Beachtung des EMV-Verhaltens", Diploma thesis TU Hamburg Harburg 2006

[6] Henry W. Ott "Noise reduction techniques in electronic systems" ISBN 0-471-85068-3

[7] H. Pfeffer, et al. "The TESLA 5 MW modulator", Fermi National Accelerator Laboratory, Batavia IL

[8] Mike Thout, lecture at DESY and personal discussion about EMI-issues of cables

[9] U. Unger, “Theorie der Leitungen", Hüthig Buch Verlag Heidelberg, 1991, ISBN 3-7785-2009-1 